\title{
Research Methodology for Real-time Stress Assessment of Nurses
}

\author{
M. Milosevic, E. Jovanov, and K. Frith \\ University of Alabama in Huntsville \\ Computers Informatics Nursing, 31(12), pp. 615-621, 2013. PMID: 24113163.
}

\begin{abstract}
This paper presents a research methodology for analysis of stress effects and allostatic load of nurses during daily activities. Stress related health issues are particularly critical in healthcare workers, in particular nurses. Typical causes of stress include inadequate staffing of nurses for the number and acuity of patients, dealing with difficult patients and families, and lack of autonomy in care delivery decisions. This is all compounded by lack of recovery time while on shift, variable shifts with limited recovery time between days worked, and fatigue from dealing with difficult patients, families, and healthcare workers. Under unresolved stress, the heart rate and other vital parameters may fail to return to the baseline. This study examined the physiological responses of nurses during care on a high-fidelity patient simulation to develop a research methodology and identify physiological parameters suitable for real-time assessment of allostatic load during work. Our results demonstrated that heart rate and heart rate variability can be reliably measured using wearable sensors to assess allostatic load. During this study and our previous related work, we acquired valuable experience regarding selection and deployment of commercially available sensors, system integration, recruitment of subjects, and general research methodology. The research methodology developed and presented in this paper can be applied to a number of other applications and experimental protocols.
\end{abstract}

Keywords: allostatic load; stress; research methods; occupational health; real-time monitoring; wearable monitoring; smartphone 


\section{Introduction}

Recent technological advances in sensors, wireless networking, mobile and cloud computing allow us to fundamentally change the way health care services are delivered. These technologies provide a new set of tools that can optimize early intervention and facilitate primary, ${ }^{1}$ secondary, ${ }^{1}$ and tertiary disease prevention. Chronic conditions such as diabetes ${ }^{2,3}$ or heart failure ${ }^{4}$ can be managed more efficiently and appropriately with the utilization of smartphone technology. With the recent explosion of the number of smartphone applications and the increase in smartphone performance, a number of health monitoring and wellness applications have become available. As of April 2012, Apple's App Store had more than 13,600 health and fitness apps available for consumers. ${ }^{5}$

Wearable systems allow individuals to monitor changes in their physical parameters and adjust behaviors in response to measurements. ${ }^{6,7}$ Integrating wearable systems with information systems used by health care providers, can even alert medical professionals if immediate interventions are needed. ${ }^{2,3}$ The wearable systems can also be used for health monitoring of patients in ambulatory settings as a part of a diagnostic procedure or for supervised recovery from an acute event or surgical procedure. ${ }^{8}$

Mobile technologies can also be used to examine job stress by monitoring physiological parameters including heart rate, heart rate variability, respiratory rate, and galvanic skin resistance. ${ }^{9}$ The significance of detecting and treating stress cannot be underestimated - job stress is associated with chronic diseases including hypertension, cardiovascular disease, depression, and immune disorders. ${ }^{10,11}$ Stress is believed to be associated with nearly half of diseases and deaths in the United States. ${ }^{12}$ To date, there are few reports about methods to detect job stress early enough to intervene. However, with current technologies the 
physiological measurements can be collected concurrently as job stress occurs ${ }^{9,13,14}$ opening a new method for interventional research to reduce job stress.

A number of research efforts have focused on wearable systems for health and wellness monitoring in the last decade. Researchers at the Massachusetts Institute of Technology (MIT) Media Lab developed a research platform called MIThril ${ }^{15,16}$ - a wearable computing platform that combines body-worn sensing, computation, and networking, enabling new applications in health, communications, and information delivery. A range of wireless medical sensors capable of collecting heart rate $(\mathrm{HR})$, oxygen saturation (SpO2), electrocardiogram (ECG), and other physiological data was developed at Harvard Sensor Network Lab and named CodeBlue. ${ }^{17}$

In this paper we present a research methodology and lessons learned about real-time job stress assessment using mobile technologies. We also present our mHealth computing infrastructure for mobile health and wellness monitoring and the experimental setup in the Real-time Physiological Lab (RTPLab) at the University of Alabama in Huntsville. The system provides continuous monitoring of the physiological parameters and collects user responses about the sources of stress using short questionnaires. 


\section{Job Stress and Allostatic Load}

The Bureau of Labor Statistics reports that all occupations are exposed to stress, but nearly $25 \%$ of stress is attributed to occupations involving healthcare workers. ${ }^{18}$ Situations reported to produce stress for hospital workers, in particular nurses, are the inadequate staffing of nurses for the number and acuity of patients, schedules (length of shift, recovery time between days worked, and night shifts), lack of recovery time while on shift (being on feet all day), interruptions, inefficient hospital systems, and the absence of unlicensed personnel to assist nurses with physically demanding tasks. ${ }^{19,20}$ Psychological stress is also experienced by nurses. These include fatigue from dealing with difficult patients, families, and other healthcare providers, stress from caring for ill or dying patients, and lack of autonomy in care delivery decisions or lack of input into unit/hospital decisions. ${ }^{21}$

Allostatic load represents the long-term physiological consequences of changes in neural or neuroendocrine activity caused by stress. McEwen and Stellar introduced the term allostatic load to represent 'the wear and tear on the body' caused by exposure to repeated or chronic stress. ${ }^{22}$ Individuals marshal physiological responses to a short, stressful situation that can be seen as an increase of stress hormones (cortisol) and heart rate and a decrease of heart rate variability. Those changes are essential for adaptation, maintenance of homeostasis, and survival. After the stressful situation passes, the individual's heart rate will typically return to baseline relatively fast; however, the level of stress hormones remains elevated for 15-45 minutes. ${ }^{23}$ Under multiple stressful situations or unresolved stress, the heart rate and other vital parameters may fail to return to base line. ${ }^{22}$ In addition to repeated stress and failure to habituate, allostatic load may be caused by failure to turn off stress responses in a timely manner or inadequate physiological response that leads to compensatory hyperactivity of other mediators. Even though allostatic load is conceptualized as a long-term dysregulation of 
neuroendocrine activity, its precursors are the moment-by-moment stressful situations found in everyday life. Therefore, it is important to detect early indications of allostatic load to prevent its profound negative effects on a workers' physical and emotional well-being.

Allostatic load can be measured as imbalances in autonomic nervous system, central nervous system, neuroendocrine, and immune system activity. Many different physiological measurements can be used to detect allostatic load in workers. ${ }^{24}$ The level of stress hormones can be measured reliably from serum, salivary, and urine samples. ${ }^{22,23}$ Heart rate variability has been used as a measurement of the autonomic nervous system's response to stress ${ }^{9,25,26}$ including job stress. ${ }^{27}$ Blood pressure and blood pressure variability have also been studied as a correlate of general stress ${ }^{11,28}$ and job stress. ${ }^{29}$

\section{Study Objectives}

We used our lab setting to carefully control nursing care events, record physiological parameters of subjects, and analyze data to answer our research question: Can allostatic load be assessed using a wearable, non-invasive physiological monitoring? 


\section{Research Methodology}

Our preliminary study involved nursing student volunteers during clinical preparation using simulations in the Learning Resource Center (LRC) at the University of Alabama in Huntsville. We used an experimental design with repeated measures and followed a predefined protocol. The experimental protocol was approved by the University IRB committee. Our power analysis revealed that we needed only 15 subjects with at least 137 observations. Students were very interested, and we had more than enough volunteers for experiments. The subjects enrolled in the experiment by using an online registration system that prevented duplicate appointments for subjects.

\section{Real-Time Physiological Monitoring Lab and the Wearable Physiological Monitoring}

The RTPLab is collaboratively run by the College of Engineering and College of Nursing, and it is located in the Learning Resource Center. The Lab allows a comprehensive evaluation of activity of the cardiovascular and autonomic nervous systems using state-of-the-art sensors and monitoring equipment. The Lab integrates a beat-by-beat non-invasive blood pressure monitor, bedside monitor, multiple physiological monitors, and other wireless sensors.

Beside the numerous physiological sensors capable of recognizing a number of physiological signals, the Lab is also equipped with high fidelity patient simulator and functional headwalls with simulated oxygen and suction, Figure 1. Details about the integrated equipment are presented in Table 1. 
Figure 1. Real-Time Physiological Monitoring Lab

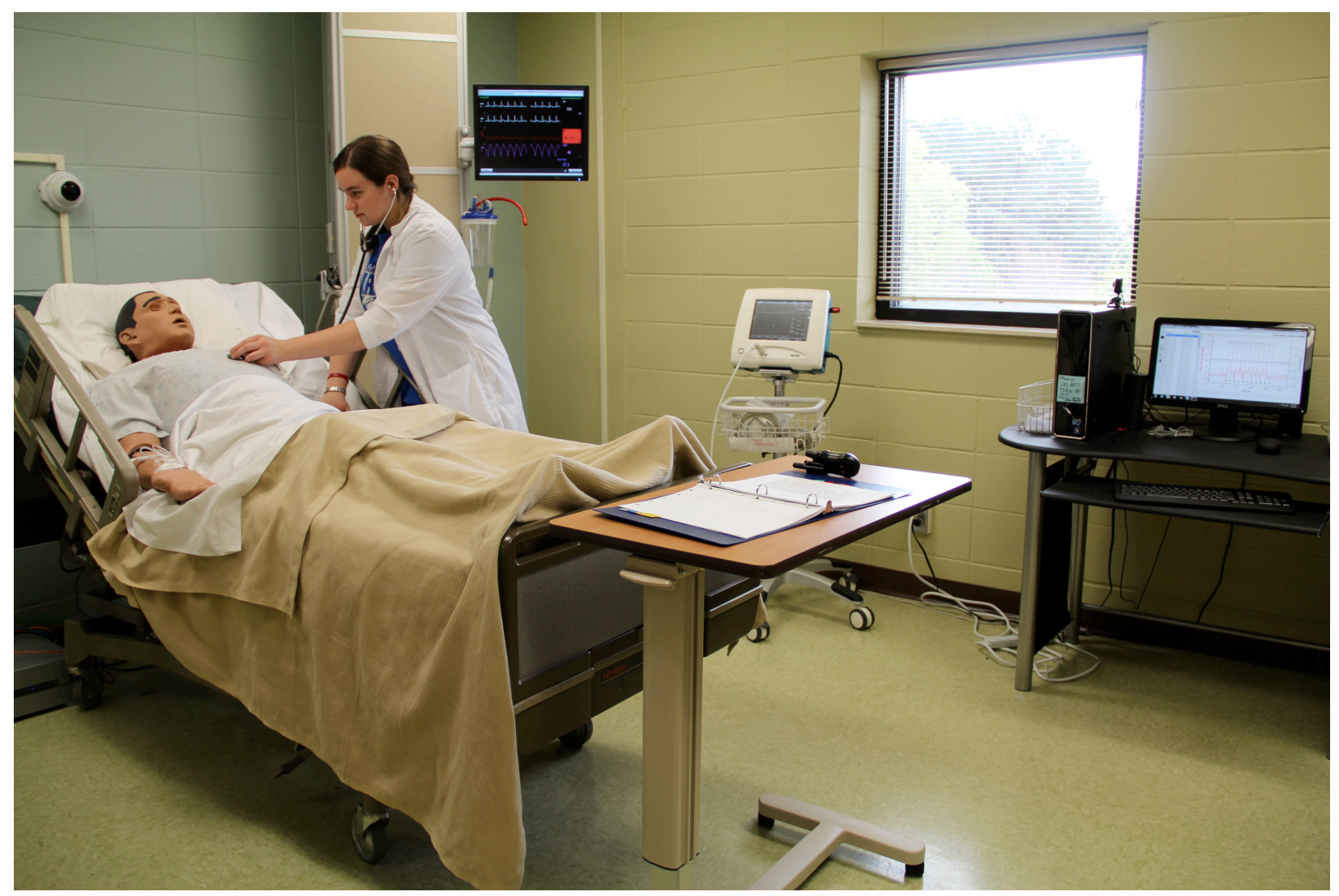

The Lab provides a unique test-bed for collaborative research and interdisciplinary education at the University. The Lab facilitates research in the field of sensor and algorithm development, simulation and modeling, psychology/human factors, information systems, healthcare management, and other interdisciplinary collaborative projects.

\section{mHealth Infrastructure}

The experimental protocol, sensors integration, data acquisition and storage rely on our mHealth computing infrastructure. The infrastructure is designed as 3-tiered architecture with wireless body area sensor networks and other aforementioned physiological monitors at Tier 1 , personal computing devices at Tier 2, and mHealth servers at Tier 3, Figure 2. 
Figure 2. Data flow in mHealth

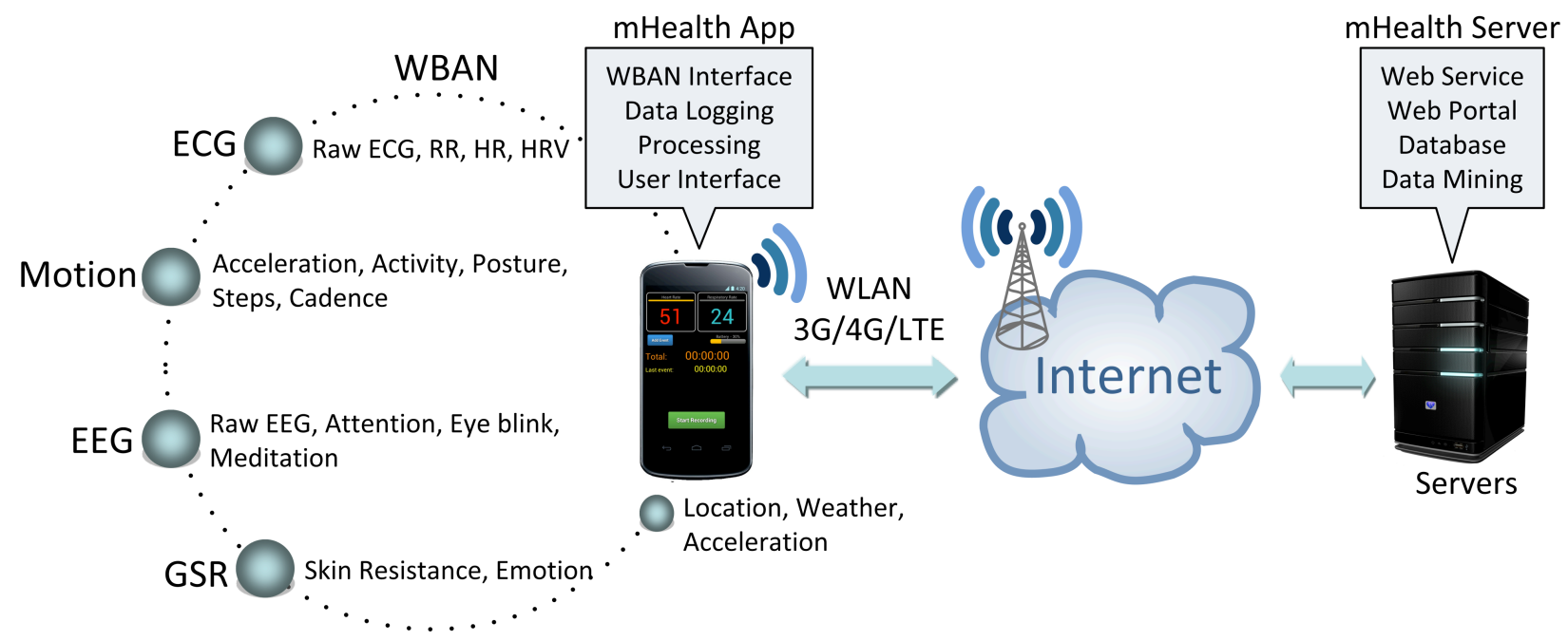

We use several different types of personal devices on Tier 2 of the mHealth infrastructure including personal computers (PC) and smartphones. We developed several applications for these personal devices, and each of them is capable of receiving and processing data from subset of physiological sensors from Tier 1 of the mHealth architecture..$^{30-32}$

A group of servers providing physiological data storage, access, and support for data mining, forms Tier 3 of the mHealth infrastructure. Servers are running a free operating system, designed to operate as virtual machines that provide portability and expandability. Physiological records are stored in an open source database. The database is specifically designed to support efficient storage of variety physiological records and record annotations, with references to de-identified subjects and equipment used to collect records. Records can be organized by experiment, and each record is precisely time-stamped, which allows global time synchronization of records. 
For processing and analysis of collected physiological records we used processing software for scientific computation. We developed a set of procedures for importing, processing, visualization, and analysis of physiological data. Because our study included multiple subjects, it was important that processing and visualization of data was standardized across all subjects. For the statistical analysis of physiological records we used statistical software. Details about the software are presented in Table 1.

\section{Guided Experimentation with Automatic Event Annotation}

Many experiments require subjects to follow precisely defined protocols. In order to facilitate and automate these types of experiments, we developed a custom PC application that performs subject guidance with a predefined experimental protocol and collects synchronized data from physiological monitors. After a protocol is defined and the experiment is started, users can see their current activity, the next activity, and the number of seconds until the next activity starts. Subjects receive an audio cue before the next activity in the experiment. Example of the user interface that guide user is shown on Figure 3 a. The application, furthermore, automatically annotates collected records in order to facilitate data analysis and processing. Time-stamped records and annotations are stored on our mHealth database server.

\section{Video Synchronization with Physiological Records}

During data analysis, it is often hard to find the exact reason for changes in physiological parameters, such as an unexpected change in heart rate. With intention to address this issue and facilitate data analysis of physiological records, we developed a PC application capable of collecting data from wearable monitors and video recordings simultaneously. The application synchronizes a video stream with physiological signals and allows synchronous replay of video and physiological recordings, Figure $3 \mathrm{~b}$. 
Figure 3. Custom PC application a) Subject guidance with a predefined experimental protocol

b) Synchronous replay of video and physiological recordings

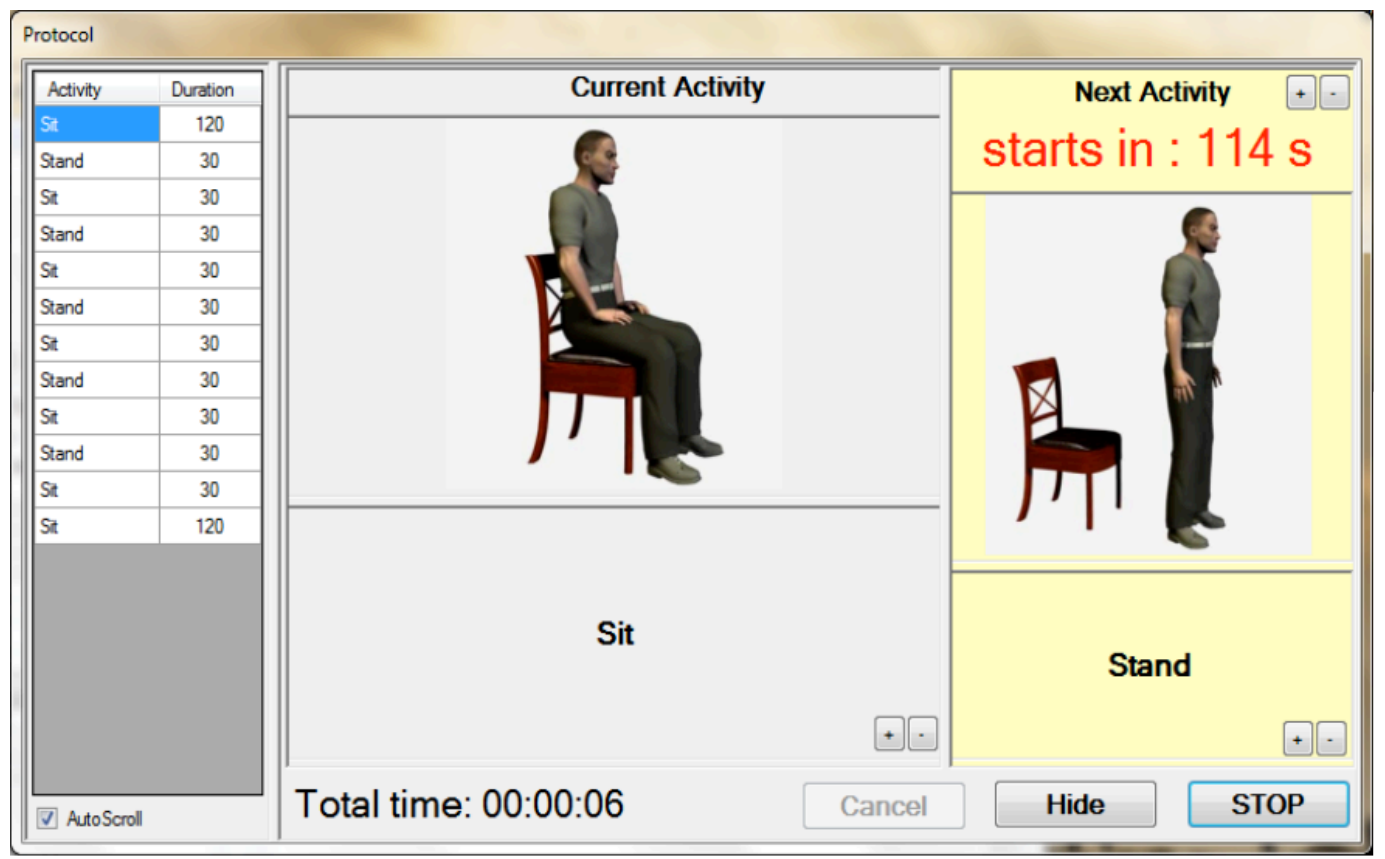

a)

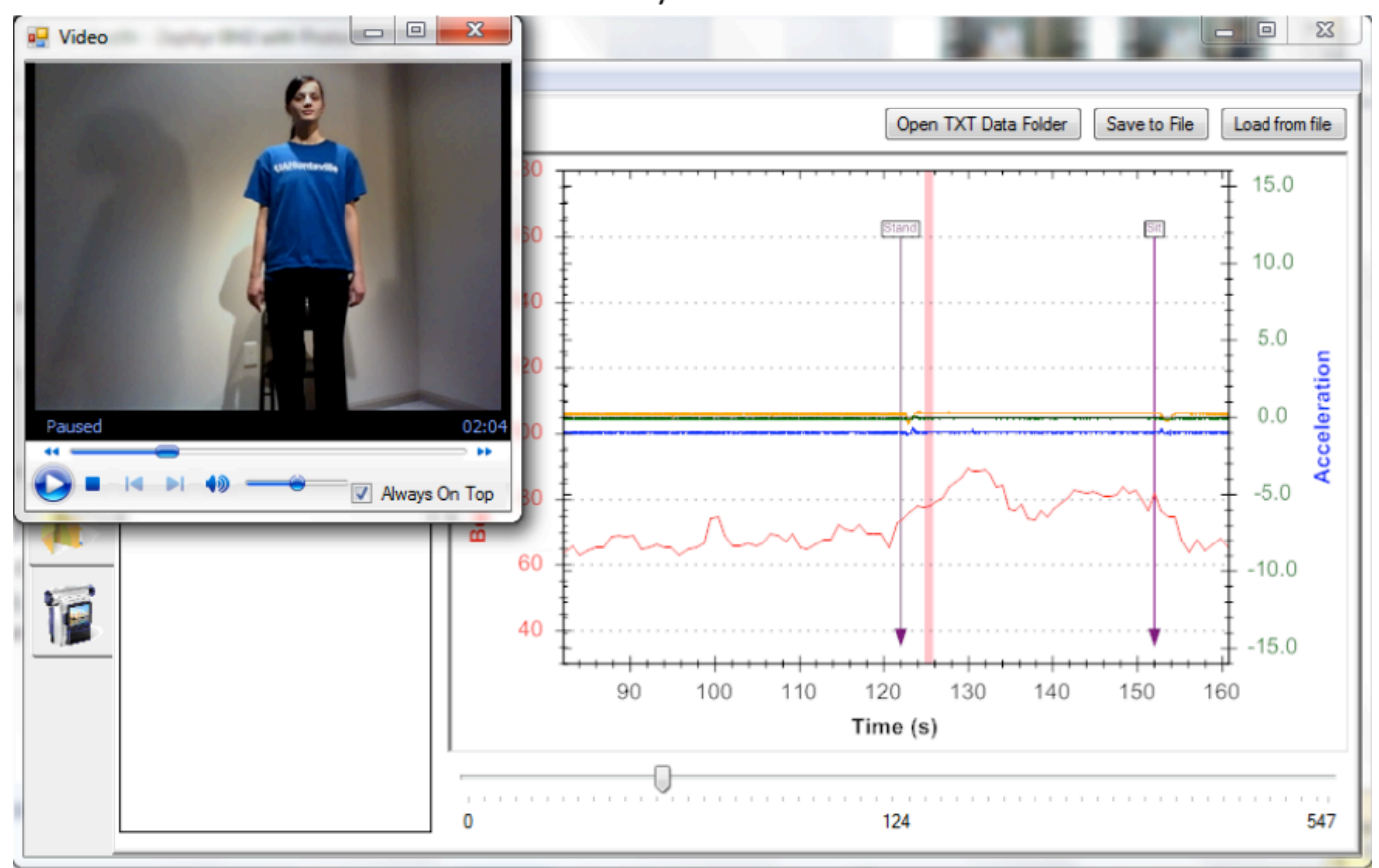

b) 


\section{Experimental setup}

We used a control room with a one-way mirror and a private patient room in the RTPLab to monitor subjects during training. From the control room, we ran the simulated nursing activity, and using our custom software synchronously recorded the activity of the subject from a video camera and their physiological signals from a physiological monitor, placed as chest belt. All records of the physiological signals were automatically uploaded to the mHealth server. The records contained interbeat intervals, heart rate, breathing rate, and acceleration on chest belt. A nursing student during a training session on the high fidelity patient simulator in the Real-time Physiological Monitoring Lab is shown on the Figure 1.

\section{Simulation protocol}

We developed a simulation that required subjects to care for a patient with a tracheostomy used for breathing. We used a computer program to run the simulation in exactly the same order for each subject. The simulation protocol ran for 30 minutes for every subject and included the following activities:

- Measurement of subject's heart rate, heart rate variability, and respiratory rate at rest for 5 minutes

- Reading "change of shift report" about patient's status

- Assessment of the patient by the subject

- Disturbance \#1: Telephone call to create an interruption during assessment

- Documentation of assessment findings by the subject

- Disturbance \#2: Telephone interruption during documentation - request for pain medication 
- Sudden respiratory distress of patient (coughing, difficulty breathing, low oxygen saturation, elevated heart rate and blood pressure) requiring urgent intervention by the subject

- Disturbance \#3: Family member apprehension and questioning of subject's actions

- End of simulation when subject pre-oxygenates and suctions patient to relieve mucous plug

- $\quad$ Five minute measurement of physiological parameters at rest. 


\section{Results}

Three particular events from the aforementioned simulation protocol were expected to be stressful for the subjects: two interruptions by telephone call (Disturbances \#1 and \#2) and sudden respiratory distress of patient. We used repeated measures analysis of variance to evaluate changes in physiological data before, during, and after training session, as well as instant changes caused by three potentially stressful events.

The training session created significant strain on subjects. On average, heart rate increased $16.7 \%$, from 82.8 to $96.6 \mathrm{bpm}(\mathrm{p}<0.001)$, falling to a slightly increased level after the training session $(84.9 \mathrm{bpm})$. All subjects had increased heart rate even before the training session that can be attributed to anxiety in anticipation of the session. The prolonged effects of the stress are visible in higher heart rate and lower heart rate variability (e.g. Root Mean Square of Successive RR Differences decreased from $38.9 \mathrm{~ms}$ to $37.7 \mathrm{~ms}$ ). The breathing rate increased also during the simulation from 16.9 to 17.7 breaths/min

The ratio of the low-frequency and high-frequency components of the RR spectrum (LF/HF) significantly changed during the simulation from 2.16 to $4.68(p<0.001)$ and followed the same pattern as heart rate - increased during the simulation and slowly return to normal after the session $2.4(p<0.001)$.

The sudden respiratory distress of simulated patient created significant physiological responses in subjects. The average heart rate increased $17.4 \mathrm{bpm}(p<0.001)$ at the onset of the event. Disturbances also significantly influenced physiological parameters by increasing demands in an already stressful situation. The first telephone call increased the heart rate on average $9 \mathrm{bpm}$ $(p<0.001)$, while the second call increased the heart rate $8.6 \mathrm{bpm}(p<0.001)$. 
Our analysis demonstrated that even though the simulation was short, there were significant changes between the baseline and ending recordings, demonstrating support for the concept of allostatic load. Detailed analysis from the study can be found in our previous work. ${ }^{33}$ 


\section{Discussion and Conclusion}

The study described in this paper examined the physiological responses of nurses during care on a high-fidelity patient simulator to develop research methodology and identify the best physiological parameters suitable for real-time assessment of allostatic load during work.

Our results indicate that physiological parameters extracted from wearable monitors, such as heart rate, heart rate variability (RMSSD, LF/HF), breathing rate, and physical activity provided a reliable indication of stress-induced physiological changes. Our findings are consistent with previous research showing that heart rate, heart rate variability, and breathing rate are measures of the short-term cardiovascular responses to stressful situations such as patient in crisis.$^{10,26,27}$

The important contribution of this study is assessment of physiological changes caused by work-place related disturbances, such as telephone calls. The disturbances created significant short-term cardiovascular responses that contribute to overall allostatic load. Lackner and Goswanmi reported similar changes when researchers gave instructions during a math task as the stress source.$^{34}$ Interruptions are particularly important if we consider the number of work related interruptions. In a typical nursing work environment, there are approximately 30 interruptions per shift, ${ }^{35}$ with numerous interruptions during drug administration. ${ }^{36}$

Continuous real-time monitoring also provides quantification of allostatic load using the dynamics of physiological changes with a 'new normal' of heart rate and heart rate variability after the stress. Because wearable physiological monitoring can be used during work and leisure, we can track long term changes of the selected parameters to examine allostatic load.

During this study and our previous related work, we acquired valuable experience regarding selection and deployment of the commercially available sensors, system integration, recruitment 
of subjects, and general research methodology. Although our experiments were focused on analysis of stress and allostatic load of nurses, the research methodology we developed and presented in this paper can be easily applied to a number of other applications and experimental protocols.

The wearable sensors were effective in capturing physiological states of subjects during their daily activities. However, we discovered that most of the commercially available fitness-grade sensors have severe limitations for research applications, such as data averaging, low resolution, forced power down modes for periods of inactivity, etc. Although many users, ourselves included, are attracted to their availability and low-cost, limitations of devices may become obvious in the later stages of the project and cause failure of an entire project. Therefore, it is crucial to thoroughly investigate and test limitations of the selected sensor technology before the beginning of a project.

Some of the commercial sensors demonstrated poor contact of belts with the skin, particularly in the case of small female subjects. In some cases, the belt would be too loose even when the smallest available belt was used. However, conductive gel proved useful for improvement of signal capture.

Traditionally, physiological monitors feature proprietary protocols and data formats with little or no support for large system integration. An increasing number of companies now provide support for integration and open data formats. However, integration of those sensors requires significant engineering effort and skills that span several programming languages, physical interfaces, and computing platforms. Since the commercially available systems provide limited support for this type of system integration, we had to develop our own support for wearable physiological monitoring and software integration of multiple sensor streams from a variety of 
sensors. Multidisciplinary collaboration was crucial for project success, and included nursing experts, engineers, and computer scientists.

One of the unexpected findings during the experiment was interference of other wireless devices with our sensors in some experimental setups. A number of different wireless protocols, such as Bluetooth, WiFi, ANT+, and cordless phones, share the same frequency band around $2.4 \mathrm{GHz}$. Their interference is acceptable in the case of a small number of devices, but becomes a serious issue in the presence of a large number of devices during events such as disaster simulation training. Consequently, we modified our software to provide reliable record collection using on-sensor storage as a backup in the case of interference.

Our experiments confirmed the importance of synchronization and efficient annotation of physiological records. Physiological records might be recorded on several devices with different internal clocks and latency, which requires precise synchronization. Moreover, behavioral cues are very important for proper interpretation of records. Therefore, we recorded video of the experiment synchronized with physiological records and combined them with manual real-time annotation by the investigators in the same monitoring application. This is another example of the custom software developed to support the needs of the research methodology.

Because we had to coordinate our study with other activities in the University Learning Resource Center, efficient management of available time slots and subjects registration was necessary. After we established available time slots for the study, we created an online form for subject registration with automated time slot management. This helped to avoid overlap of subjects in the same period, which was important to remove exposure of subjects to preceding simulations and study protocol. The registration system also reduced the wait times for subjects. 
Sufficient number of investigators for the study is always important, so planning has to be performed carefully. Because our custom software helped us to reduce the number of investigators, the study was possible with four investigators (three actively involved and one as backup). We used one investigator to run the simulation on the high fidelity patient simulator, the second placed sensors on subjects and created artificial disturbances described in protocol of the study, while the third investigator annotated significant events using custom developed software.

As mentioned earlier, we developed a set of custom scripts for data processing and analysis. Although custom scripts required significant development time, the scripts facilitated faster and flexible data analysis. This proved particularly useful for the preparation of data in custom formats for statistical analysis packages performed by the statistics expert in our team. Some analyses required a very specific organization of data sets, which could be done much more efficiently using custom scripts in the native database environment.

Future work includes long-term wearable monitoring (weeks instead of hours), automatically triggered self-reports, and the development of personalized assessment of the allostatic load. Monitoring for extended periods will provide data for long-term trends and model development. Self-reported questionnaires triggered by the peak allostatic load on smartphones will facilitate collection of data regarding the perceived level and causes of stress in specific work settings.

The real-time assessment of allostatic load can be used to provide real-time alerts for nurses to engage in stress reducing activities. Objective assessment of the allostatic load can be also used by the hospital administrators to understand job stress in their own settings and adjust nurse staffing or patient assignments to improve quality of patient care and quality of life for nurses. 


\section{References}

1. Luxton D, McCann R, Bush N, Mishkind M, Reger G. mHealth for mental health: integrating smartphone technology in behavioral healthcare. Professional Psychology: Research \& Practice. 2011;42(6):505-512. doi:10.1037/a0024485.

2. Frøisland $D$, Arsand $E$, Skårderud F. Improving diabetes care for young people with type 1 diabetes through visual learning on mobile phones: mixed-methods study. Journal of Medical Internet Research. 2012;14(4):e111-e111.

3. Milosevic M, Shrove MT, Jovanov E. Applications of Smartphones for Ubiquitous Health Monitoring and Wellbeing Management. Journal of Information Technology and Applications. 2011;1(1):7-15.

4. Blake H. Innovation in practice: mobile phone technology in patient care. British Journal of Community Nursing. 2008;13(4):160.

5. Dolan B. An Analysis of Consumer Health Apps for Apple's iPhone 2012. Mobi Health News Available at: http://mobihealthnews.com/17925/just-launched-our-2012-consumer-health-appsreport/. Accessed October 22, 2012.

6. The smartphone will see you now. Harvard Heart Letter. 2011;22(2):3-3.

7. Digifit. Running \& FitNews. 2012;30(4):3-3.

8. Jovanov E, Milenkovic A, Otto C, de Groen P. A wireless body area network of intelligent motion sensors for computer assisted physical rehabilitation. Journal of NeuroEngineering and Rehabilitation. 2005;2(1):2-6.

9. Weippert M, Kumar M, Kreuzfeld S, Arndt D, Rieger A, Stoll R. Comparison of three mobile devices for measuring R-R intervals and heart rate variability: Polar S810i, Suunto t6, and an ambulatory ECG system. European Journal of Applied Physiology. 2010;109:779-786.

10. Sun J, Wang S, Zhang J-Q, Li W. Assessing the cumulative effects of stress: The association between job stress and allostatic load in a large sample of Chinese employees. Work \& Stress. 2007;21(4):333-347.

11. McNeely E. The consequences of job stress for nurses' health: time for a check-up. Nursing Outlook. 2005;53(6):291-299.

12. Quick JC. Introduction to the measurement of stress at work. Journal of Occupational Health Psychology. 1998;3(4):291-293.

13. Jovanov E, Frith K, Anderson F, Milosevic M, Shrove MT. Real-time monitoring of occupational stress of nurses. Conf Proc IEEE Eng Med Biol Soc. 2011;2011:3640-3643. doi:10.1109/IEMBS.2011.6090612.

14. Jovanov E, Lords A, Raskovic E, Cox P, Adhami R, Andrasik F. Stress Monitoring Using Distributed Wireless Intelligent Sensor System: Quantifying Stress Levels Based on Measures 
of Heart-Rate Variability (HRV) Using Reliable, High-Precision Instrumentation and Synchronized Measurements. IEEE Engineering in Medicine and Biology Magazine. 2003;May/June(49-55).

15. DeVaul RW, Schwartz SJ, Pentland A "Sandy." MIThril: context-aware computing for daily life. MIT Media Lab; 2001.

16. Pentland A. Healthwear: medical technology becomes wearable. Computer. 2004;37:42-49. doi:10.1109/MC.2004.1297238.

17. Malan D, Fulford-jones T, Welsh M, Moulton S. CodeBlue: An ad hoc sensor network infrastructure for emergency medical care. In: In International Workshop on Wearable and Implantable Body Sensor Networks. Boston, MA, USA; 2004.

18. Bureau of Labor Statistics. Occupational Injuries and IIInesses and Fatal Injuries Profiles. Available at: http://data.bls.gov/gqt/InitialPage. Accessed September 15, 2012.

19. Mion LC, Hazel C, Cap M, Fusilero J, Podmore ML, Szweda C. Retaining and recruiting mature experienced nurses: a multicomponent organizational strategy. Journal of Nursing Administration. 2006;36(3):148-154.

20. National Institute of Occupational Safety and Health. Exposure to Stress: Occupational Hazards in Hospitals (2008-136). Available at: http://www.cdc.gov/niosh/docs/2008-136/. Accessed September 15, 2012.

21. Santos SR, Carroll CA, Cox KS, et al. Baby boomer nurses bearing the burden of care: a four-site study of stress, strain, and coping for inpatient registered nurses. Journal of Nursing Administration. 2003;33(4):243-250.

22. McEwen BS. Stressed or stressed out: what is the difference? Journal of Psychiatry \& Neuroscience. 2005;30(5):315-318.

23. Looser RR, Metzenthin P, Helfricht $S$, et al. Cortisol is significantly correlated with cardiovascular responses during high levels of stress in critical care personnel. Psychosomatic Medicine. 2010;72(3):281-289.

24. Collins SM. Emerging methods for the physiological assessment of occupational stress. Work: Journal of Prevention, Assessment \& Rehabilitation. 2001;17(3):209-220.

25. Negoescu R, Dinca-Panaitescu S, Filcescu V, lonescu D, Wolf S. Mental stress enhances the sympathetic function of QT variability in an RR-independent way. Integrative Physiological \& Behavioral Science. 1997;32(3):220-227.

26. Ottaviani C, Shapiro D, Davydov DM, Goldstein IB. Autonomic stress response modes and ambulatory heart rate level and variability. Journal of Psychophysiology. 2008;22(1):28-40.

27. Collins MA. The relation of work stress, hardiness, and burnout among full-time hospital staff nurses. Journal of Nursing Staff Development. 1996;12(2):81-85. 
28. Buerhaus PI, Donelan K, Ulrich BT, Norman L, Dittus R. State of the Registered Nurse Workforce in the United States. [Article]. Nursing Economics January/February. 2006;24(1):612.

29. Gallo LC, Bogart LM, Vranceanu A-M, Walt LC. Job Characteristics, Occupational Status, and Ambulatory Cardiovascular Activity in Women. Annals of Behavioral Medicine. 2004;28(1):62-73.

30. Milosevic M, Shrove MT, Jovanov E. Applications of Smartphones for Ubiquitous Health Monitoring and Wellbeing Management. Journal of Information Technology and Applications. 2011;1(1):7-15.

31. Shrove MT, Milosevic M, Johnson B, Milenkovic A, Jovanov E. iCareWell: Real-time Wellness Monitor. In: Boston, MA, USA; 2011:24.

32. Jovanov E, Milosevic M, Milenkovic A, Shrove MT, Frith K, Anderson F. Personalized Assessment of Occupational Stress of Nurses. In: Washington, DC; 2011.

33. Milosevic M, Jovanov E, Frith, KH, Vincent J. Preliminary Analysis of Physiological Changes of Nursing Students during Training. IEEE Engineering in Medicine and Biology Society. 2012;34th Annual International Conference.

34. Lackner HK, Goswami N, Hinghofer-Szalkay H, et al. Effects of stimuli on cardiovascular reactivity occurring at regular intervals during mental stress. Journal of Psychophysiology. 2010;24(1):48-60.

35. Cornell P, Riordan M, Townsend-Gervis M, Mobley R. Barriers to Critical Thinking: Workflow Interruptions and Task Switching Among Nurses. JONA: The Journal of Nursing Administration. 2011;41(10):407-414. doi:10.1097/NNA.0b013e31822edd42.

36. Anthony K, Wiencek C, Bauer C, Daly B, Anthony MK. No Interruptions Please: Impact of a No Interruption Zone on Medication Safety in Intensive Care Units. Crit Care Nurse. 2010;30(3):21-29. doi:10.4037/ccn2010473. 
Figure and Table Legend 